Int. J. Dev. Biol. 49: 909-914 (2005)

doi: $10.1387 /$ ijdb.052017at

Original Article

\title{
Mouse chimaeras developed from electrofused blastocysts: new evidence for developmental plasticity of the inner cell mass
}

\author{
ANDRZEJ K. TARKOWSKI*, KAMILA JAGIELLO, RENATA CZOLOWSKA and WACLAW OZDZENSKI \\ Department of Embryology, Institute of Zoology, Faculty of Biology, Warsaw University, Warsaw, Poland
}

\begin{abstract}
Blastocysts obtained from mice differing in pigmentation (albino versus pigmented) and the isoforms of glucose phosphate isomerase (GPI 1A versus 1B) were electrofused and those containing a single chimaeric inner cell mass (ICM) were transferred to the uterus of pseudopregnant recipients. The pups were recovered on the $20^{\text {th }}$ day by Caesarian section and fostered by females that had littered on the previous night or $\mathbf{2 4} \mathrm{h}$ earlier. Altogether nine adult animals and two pups, which died soon after delivery, were available for GPI analysis. Between 9 and 13 organs/ tissues were examined and the relative contribution of the GPI 1A and 1B isoforms was estimated using an electrophoretic GPI assay. Eight adult animals were overtly chimaeric and one was chimaeric in some internal tissues only. Eight mice were males: seven were fertile, one was infertile. The ninth adult mouse was a hermaphrodite. The fertile animals produced sperm of one genotype only, i.e. derived either from the albino or from the pigmented component. This is the first report showing that adult chimaeras can be produced from two combined blastocysts, provided that fusion of the adhering trophectoderm cells is first induced and the orientation of blastocysts enables the two ICMs to integrate into a single ICM. Our results suggest that in the preimplantation blastocyst, the organisation of the ICM remains labile thus making it possible for the fused blastocysts to establish new embryonic organisation and to develop into a single organism.
\end{abstract}

KEY WORDS: mouse, blastocyst, electrofusion, inner cell mass, chimaera

\section{Introduction}

The mammalian egg is radially symmetrical around the animalvegetal axis. Site of the extrusion of the polar bodies designates the animal pole of this axis. In the mouse, the early blastocyst is radially symmetrical around the embryonic-abembryonic axis, but at a certain stage of expansion, it becomes bilateral and according to Gardner (1997) the axis of symmetry of the embryo is aligned with the animal-vegetal axis of the egg. Smith $(1980,1985)$ was the first to observe the bilateral symmetry of a mouse blastocyst at the time of implantation. However, Gardner (1997) showed that signs of bilaterality appear already in the preimplantation blastocyst. Thus the question arises what is the developmental significance of the morphological signs of bilateral symmetry, i.e. do they indicate that the determination of the blastocyst's organisation has already begun? One of the possible experimental approaches to try to answer this question is to construct chimaeric blastocyst in which the inner cell mass (ICM) would be composed of two inner cell masses derived from two separate blastocysts and to see if thus produced embryo will develop normally. Our present study shows that two blastocysts combined together by an electrofusion (Ozdzenski et al., 1997) are able to form a blastocyst containing a single chimaeric ICM. Such blastocyst is able to develop into one foetus and eventually into one chimaeric adult. These results suggest, though do not definitely prove, that the postulated bilateral symmetry of mouse blastocyst remains labile up to the implantation stage.

\section{Results}

In our fusion experiments the effectiveness of producing chimaeric blastocysts from two aggregated blastocysts was $48 \%$ (Table 1). No correlation was observed between various spatial configurations of the two aggregated blastocysts (Fig. 1) and the overall frequency of fusions and the frequency of blastocysts with

Abbreviations used in this paper: BSA, bovine serum albumin; GPI, glucose phosphate isomerase; ICM, inner cell mass; PBS, phosphate buffered saline.

\footnotetext{
*Address correspondence to: Dr. Andrzej K. Tarkowski. Department of Embryology, Institute of Zoology, Faculty of Biology, Warsaw University, Miecznikowa 1, 02-096 Warsaw, Poland. Fax: +48-22-5541-210 or 203. e-mail: akt@biol.uw.edu.pl
} 
A

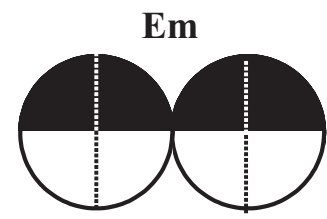

Ab
B

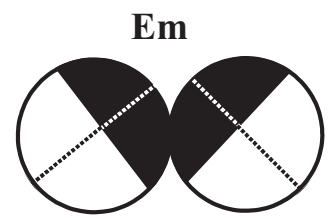

Ab

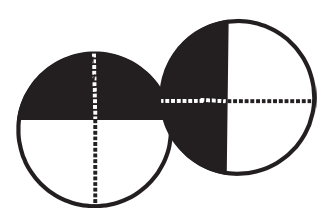

Ab
$\mathbf{A b}$

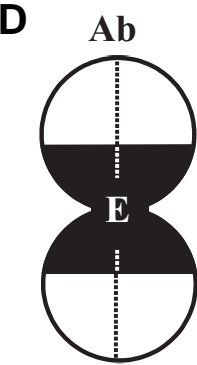

Ab
Fig. 1. Schematic representation of four spatial arrangements of blastocysts aggregated in preparation for electrofusion. Embryonic-abembryonic ( $E m-A b)$ axes can be paralle/ (A), oblique (B), perpendicular (C) or reverse (D) to each other. Dotted lines designate embryonic-abembryonic axis, Em lor E)-embryonic pole, Ab-abembryonic pole. single ICM (Figs 2A and $3 \mathrm{~A}$ ) versus blastocysts with two ICMs (Figs 2B and $3 \mathrm{~B}$ ). The blastocyst with two ICMs forms when the polar trophectoderms in the region of adhesion fail to fuse thus preventing the two ICMs to incorporate into a single ICM. Almost $80 \%$ of transferred blastocysts with one ICM underwent successful implantation and 11 out of 15 implanted embryos developed successfully to term. All recovered young were placed with lactating foster mothers and nine of them survived to adulthood.

While distinction of blastocysts with two ICMs was simple, this was not always the case with those with one ICM. When examining live blastocysts, it was sometimes hard to determine whether the two ICMs fused completely into a single structure, or whether they were positioned in very close proximity. In the latter case, they could, perhaps, develop into two independent egg cylinders (and later into two foetuses), as do blastocysts with clearly separated two ICMs (Ozdzenski et al., 1997; our unpublished results). To get better understanding of the architecture of chimaeric blastocysts in general and of those with one ICM in particular, we fused blastocysts originating from eggs injected with dextran conjugated with two different fluorochromes and after fixation, examined them with the confocal microscope. One of such blastocysts (classified, when observed alive, as having one ICM) is shown in Fig. 3A. The boundary between the two populations of cells (originating from two separate blastocysts) was still visible, suggesting that cell intermingling has not yet begun on a large scale. In the mural trophectoderm of this blastocyst the border between the two trophectoderms was clearly delineated along the whole circumference of the embryo. The demarcation of the boundary between the two original blastocysts was even more visible in the blastocysts with two ICMs (Fig. 3B).
Chimaerism of the born animals was estimated on the basis of coat pigmentatation (Figs 4,5) and the type of GPI isoforms present in several examined organs and tissues (Table 2). The two young that were found dead $24 \mathrm{~h}$ after birth, had pigmented eyes and all examined organs (brain, eyes, heart, lungs, liver, intestine, kidneys, skin and muscles) contained only the $1 \mathrm{~B}$ isoform of GPI, characteristic of the F1 blastocyst. These two individuals were thus non-chimaeric. In nine experimental adult animals 10 to 13 organs or tissues were subjected to GPI analysis. One animal (JW5/2 in Table 2) was not an overt chimaera (fully albino) and chimaerism was detected only in the intestine, liver and both adrenal glands (with very low level of $1 \mathrm{~B}$ isoform). Other eight animals proved to be chimaeric and the majority of their tested tissues contained both isoforms of GPI. In general, the contribution of the two components, $1 \mathrm{~A}$ (albino) and $1 \mathrm{~B}(\mathrm{~F} 1$, pigmented), as judged by the contribution of the two GPI isoforms, corresponded to their contribution to the coat pigmentation (animals assigned 'JW' in Table 2 and Fig. 4 are arranged from left to right in the sequence of the increasing pigmentation in the coat).

Analysis of coat pigmentation showed that there were no special features in the pattern of coat pigmentation, that would distinguish chimaeras obtained by blastocyst fusion from those produced, routinely, in this and other laboratories, by aggregation of cleaving embryos. Even in such a small sample of animals as those shown in Fig. 4, the pattern of coat pigmentation varied from «pure» albino, through intermediate types, to nearly completely pigmented.

Amongst nine adults one was a hermaphrodite (Fig. 5) with a small testis on the left side and an ovary, associated with the uterine horn filled with fluid, on the right side. The remaining eight

TABLE 1

GENERATION OF CHIMAERIC BLASTOCYSTS AND THEIR FURTHER SURVIVAL AFTER TRANSFER TO RECIPIENT FEMALES

Number of pairs of blastocysts subjected to fusion

Number (\%) of successfully fused blastocysts Postimplantation development of blastocysts with one ICM

\begin{tabular}{|c|c|c|c|c|c|c|c|}
\hline \multirow{2}{*}{85} & $\begin{array}{c}41(48.2) \\
(100.0)\end{array}$ & $22(53.7)$ & $19(46.3)$ & 19 & $\begin{array}{c}15(78.9) \\
(100.0)\end{array}$ & $\begin{array}{c}11 \\
(73.3)\end{array}$ & $\begin{array}{c}9 \\
(60.0)\end{array}$ \\
\hline & & & & & & $\begin{array}{c}2 \text { or } Q^{*} \\
8 \mathrm{O}^{\lambda} \mathrm{O}^{\star * *} \\
1 \mathrm{H}^{* *}\end{array}$ & $\begin{array}{c}8 \widehat{O}^{\lambda * *} \\
1 \mathrm{H}^{* *}\end{array}$ \\
\hline
\end{tabular}

Symbols: *, non-chimaeric; ${ }^{*}$, chimaeric; $\mathrm{H}$, hermpahrodite 
animals were males. The males were crossed with albino females. One male was considered to be infertile as none of several females with which it had mated became pregnant. Of these females, three were sacrificed on the day of vaginal plug. Spermatozoa were occasionally observed in smears of uterine fluid, but none of the ovulated eggs were fertilised. The remaining seven males produced several litters. Progeny, ranging in number between 44 and 124, was, as a rule, of one genotype only, i.e. either albino or pigmented, thus suggesting that the germ line was nonchimaeric.

\section{Discussion}

In this study we have succeeded in producing, for the first time, chimaeric mice by electrofusion of two blastocysts into one blastocyst containing single ICM derived from two separate ICMs. Permanent integration of two blastocysts requires induction of cell fusion between the trophectoderm cells of the two adhering blastocysts, which can be achieved either by the Sendai virus (Tarkowski and Wojewódzka, 1982) or electric impulses (Ozdzenski et al., 1997). The layer of thus produced 'hybrid' trophectoderm, that separates the two cavities, ultimately breaks down and retracts and the two cavities coalesce into one. Depending on the spatial orientation of the two blastocysts during aggregation/fusion, the nascent chimaeric blastocyst can have either two separate (and non-chimaeric) ICMs or only one chimaeric ICM.

Fusion of blastocysts is certainly not a method of choice for the production of a large number of chimaeric mice. It may prove useful, however, for special purposes, such as the study of the plasticity of the structural organisation of embryos at the blastocyst stage (as in the present study), or the study of the development of blastocysts with two ICMs, which resembles development of identical twins originating spontaneously from one blas-

tocyst (and known in human embryology as monochorial).

In the development of the mouse, bilateral symmetry of the embryo was first observed by Smith $(1980,1985)$ who studied implanting blastocysts. Gardner (1997) observed that the expanded, but still unimplanted mouse blastocyst, viewed from the embryonic pole, is not circular, but elliptical and consequently the blastocyst and the inner cell mass are bilaterally symmetrical.

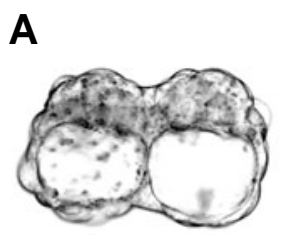

$30^{\prime}$

B

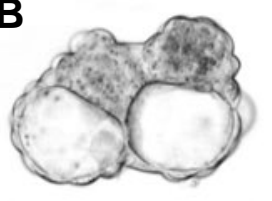

$1 \mathrm{~h}$ Bar, $100 \mu \mathrm{m}$.

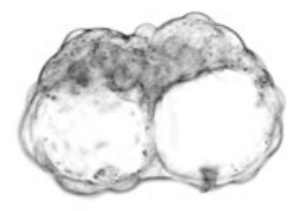

$5 \mathrm{~h}$

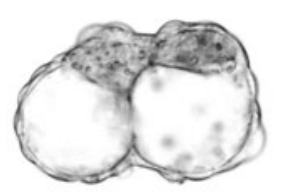

$5 \mathrm{~h}$

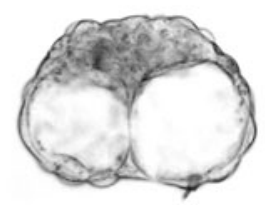

$8 \mathrm{~h}$

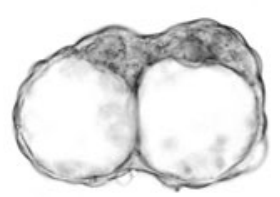

$7 \mathrm{~h}$

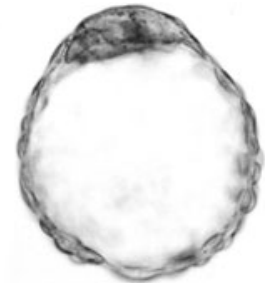

21h

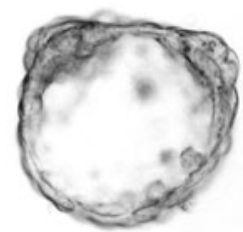

23h
Fig. 2. Fusion of pairs of blastocyts and formation of chimaeric blastocysts with one ICM (A) and with two ICMs (B). Hours and minutes shown at the bottom of figures denote time that has elapsed since application of electric pulses. The initial arrangement of both pairs was similar (Em - Ab axes parallel as in Fig. 1A), but the outcome was different and could not have been predicted for many hours after electrofusion. Fusion between adhering cells of polar and mural trophectoderm begins 30-60 min after application of electric pulses and continues during the next few hours. The septum composed of fused trophectodermal cells and separating the two cavities, stretches during expansion of blastocysts and finally breaks down and retracts which leads to the formation of one common blastocyst cavity. The blastocyst in Fig. 2B (23 h) is slightly contracted for better visualisation of the two separate ICMs.
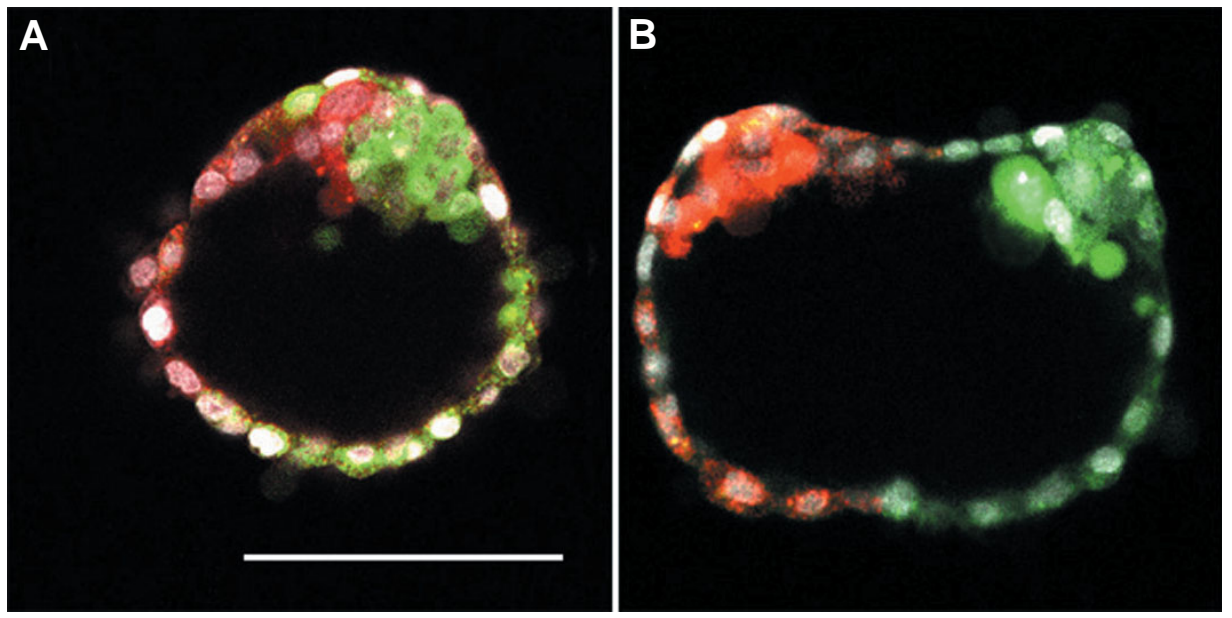

Fig. 3. Blastocysts produced by electrofusion of two blastocysts that developed from one-cell eggs injected with dextrans conjugated with fluoresceine (green) and rhodamine (red). Fixed $24 \mathrm{~h}$ after electrofusion. Confocal microscope. Bar, $100 \mu \mathrm{m}$. (A) Blastocyst with one chimaeric inner cell mass: both 'green' and 'red' cells contribute to the ICM; note that mural trophectoderm on both sides of the ICM is derived from different blastocysts. (B) Blastocyst with two inner cell masses. The sharp demarcation line is visible in the trophectoderm between the two non-chimaeric ICM.

Gardner also pointed out that the second polar body, which is located at the animal pole of the egg and designates its animalvegetal axis, in the blastocyst is situated at the boundary of the ICM and the blastocoel and designates the axis of the bilateral symmetry of the blastocyst. Does the bilateral organisation of the blastocyst enforce the bilateral symmetry of the future embryo? If the axis of bilateral symmetry and the embryonic-abembryonic 


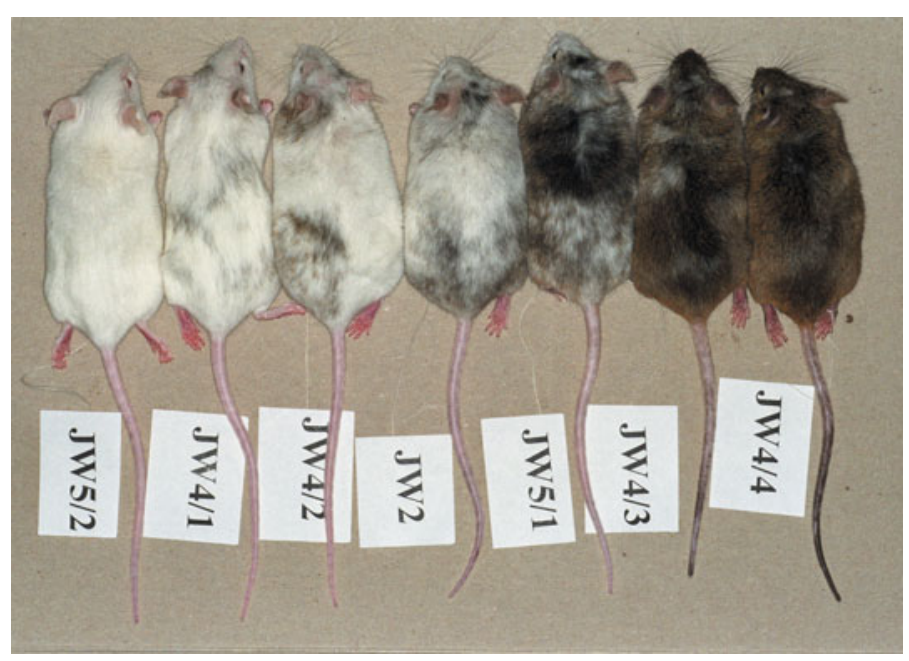

Fig. 4. Adult chimaeras (approximately 5 months old). The totally nonpigmented animal (far left) was chimaeric in some internal organs. Marking on the labels helps to identify animals in Table 2.

axis of the ICM have already been set before implantation, then any experimental interference in the ICM should have disastrous effect on further embryonic development. However, some studies suggest that at least until implantation, the ICM has great developmental plasticity. In the cattle, twins can develop from bisected blastocysts (Ozil, 1983) and from demi-blastocysts (each inheriting a part of the primary ICM) formed by herniation through the zona pellucida (Skrzyszowska et al., 1999). In the mouse, whole ICM injected into the blastocoel can merge with the native ICM and develop jointly into one chimaeric foetus/animal (Gardner, 1971; Gardner and Johnson, 1973). If one assumes that the ICM (or its part) is axially organised, then in all experiments cited above, the ICM would have to undergo a total reorganisation in order to establish new axis of symmetry and assign new developmental fates to the constituent cells. Our present study provides additional support for the belief, that in the preimplantation mouse blastocyst, the ICM is a highly regulative structure. We aggregated and fused blastocysts randomly in regards to their hypothetical axis of bilateral symmetry, so that in the majority of cases, in the «dual» ICM the two original axes must have been oriented under different angles. Moreover, the embryonicabembryonic axial orientation of the two blastocysts was sometimes reverse (cfFigs 1D). Irrespective of these postfusion rearrangements, $73.3 \%$ of implanted blastocysts with one ICM gave rise to chimaeric full term foetuses (Table 1). We cannot exclude the possibility that the remaining chimaeric embryos died because of severe axial

${ }^{4}$ hybrid band also present

$>$ dominating, >> highly dominating

n.e. not examined discordance of the two ICMs, but due to the very high rate of normal development of implanted blastocysts, this interpretation is unlikely. In conclusion, the present work shows that despite the signs of bilateral symmetry present in preimplantation mouse blastocyst, neither the postulated axis of the ICM nor the exact embryonic fate of its cells have been determined at this stage. Our work provides additional evidence of the developmental plasticity of the mouse preimplantation embryo and agrees with earlier studies, which using different experimental approaches, suggested that the structure of the preimplantation blastocyst remains undetermined.

The pattern of coat pigmentation and the genotype of internal organs/tissues, as determined by the presence of GPI isoforms, show that chimaeras generated in our experiments by the fusion of the blastocysts, closely resemble chimaeras produced by the aggregation of cleaving embryos. The high incidence $(9 / 11)$ of chimaeras in the group of animals born from the blastocysts with one «dual» ICM was similar or even higher than in experiments in which the cleaving embryos were aggregated (cf Mullen and Whitten, 1971; Mystkowska et al., 1979; see also references in: Falconer and Avery, 1978). Once a chimaeric blastocyst with one "dual» ICM is formed, the chance of it developing into a chimaera is theoretically higher than in the case of blastocyst produced by aggregation of cleaving embryos. This is because in the former type of blastocyst the ICM is 1:1 chimaeric (assuming that the two fused blastocysts had similar number of cells). In contrast, in blastocysts produced by aggregation of cleaving embryos, the allocation of the two types of cells to common ICM may be unequal and in addition, in later stages of development, one cell type may become predominant (cfFalconer and Avery, 1978).

TABLE 2

CHIMAERISM OF THE COAT, GENOTYPE OF PROGENY AND THE CONTRIBUTION OF GPI 1A AND 1B ISOZYMES TO VARIOUS TISSUES/ORGANS OF ADULT CHIMAERAS

\begin{tabular}{|c|c|c|c|c|c|c|c|c|c|}
\hline & JW5/2 & JW4/1 & JW4/2 & JW2 & JW5/1 & JW4/3 & JW4/4 & $\mathrm{K} 1$ & $\mathrm{~K} 2$ \\
\hline Coat $^{1}$ & $a$ & $a>>b$ & $a>>b$ & $a>b$ & $a<b$ & $a<<b$ & $a<<b$ & $a=b$ & $a<b$ \\
\hline Progeny $^{2}$ & a & - & a & $b$ & a & b & $b$ & - & $b$ \\
\hline blood $^{3}$ & a & $a>>b$ & $a>>b$ & $a>>b$ & $a<b$ & $a=b$ & $b$ & $a=b$ & $a=b$ \\
\hline tongue & a & $a>b^{4}$ & $a>>b^{4}$ & $a<b^{4}$ & $a<b^{4}$ & $a<<b^{4}$ & $b$ & $a=b^{4}$ & $a<<b^{4}$ \\
\hline brain & a & $a>b$ & $a=b$ & $a=b$ & $a=b$ & $a<<b$ & $a<<b$ & $a=b$ & $a=b$ \\
\hline eye & a & $a=b$ & $a>b$ & $a<b$ & $a<b$ & $a<<b$ & $a<<b$ & n.e. & n.e. \\
\hline heart & a & $a>b$ & $a>b$ & $a=b$ & $a=b$ & $a=b$ & $a=b$ & $a=b$ & $a=b$ \\
\hline lungs & a & $a>b$ & $a>b$ & $a=b$ & $a=b$ & $a<b$ & $a<<b$ & $a=b$ & $a<b$ \\
\hline intestine & $a>>b$ & $a>b$ & $a=b$ & $a=b$ & $a=b$ & $a<b$ & $a<b$ & $a=b$ & $a<b$ \\
\hline liver & $a>>b$ & $a>b$ & $a<b$ & $a<<b$ & $a<b$ & $a<b$ & $a<<b$ & $a=b$ & $a=b$ \\
\hline spleen & n.e. & $a>b$ & $a>b$ & $a=b$ & $a<b$ & $a<b$ & $b$ & $a=b$ & $a<b$ \\
\hline kidney & a & $a>b$ & $a>>b$ & $a=b$ & $a=b$ & $a=b$ & $a<b$ & $a=b$ & n.e. \\
\hline adrenal gland & $a>>b$ & $a>>b$ & $a>>b$ & $a=b$ & n.e. & n.e. & $a=b$ & n.e. & n.e. \\
\hline \multirow[t]{2}{*}{ gonad } & a & $a>>b$ & $a>>b$ & $a=b$ & $a>>b$ & $a<b$ & $a<<b$ & $\mathrm{a}=\mathrm{b}$ (ovary) & $a=b$ \\
\hline & & & & & & & & $a>b$ (testis) & \\
\hline striated muscle & a & b & $a>>b^{4}$ & $a=b^{4}$ & $a>b^{4}$ & $a=b^{4}$ & $a=b^{4}$ & $a=b$ & $a=b$ \\
\hline \multicolumn{10}{|l|}{ Total no. of examined } \\
\hline tissues/organs & 12 & 13 & 13 & 13 & 12 & 12 & 13 & 12 & 10 \\
\hline
\end{tabular}

${ }^{1}$ Coat: 'a' indicates albino fur and 'b' pigmented fur

2 Progeny: 'a' indicates albino progeny and 'b' pigmented progeny

${ }^{3}$ In this and following rows ' $a$ ' indicates 1 A GPI isoform and 'b' $1 \mathrm{~B}$ isoform

= equal proportion of albino and pigmented hair/equal contribution of both GPI isozymes, 
Similarly to the chimaeras produced by aggregation of cleaving embryos, the chimaeras constructed by fusion of blastocysts originate from two embryos selected at random in regards to their genetic sex. Therefore, one could expect, that any group of chimaeras should have $25 \%$ females $(X X \leftrightarrow X X), 25 \%$ males $(X Y \leftrightarrow X Y)$ and $50 \%$ sex chimaeras $(X X \leftrightarrow X Y)$. Animals belonging to the last group, develop predominantly into phenotypical males that produce sperm only from the male $(\mathrm{XY})$ component and rarely develop into true hermaphrodites (Tarkowski, 1961; Mystkowska and Tarkowski, 1968; reviewed by McLaren, 1976; 1984). Among nine adults produced in this study there were one hermaphrodite and eight males, seven of which produced progeny of one genotype only (one male produced sperm but was infertile). In our study, the absence of females and of $X Y \leftrightarrow X Y$ males with germ line chimaerism does not agree with expected statistical distribution of sex genotypes, but considering the low number of animals (9) in our experimental sample, this could simply happen by chance. Unfortunately we do not have any information on the genetic sex of the two components of our chimaeras.

\section{Materials and Methods}

\section{Animals}

Animals were kept under $14 \mathrm{~h}$ light/ $10 \mathrm{~h}$ dark lighting regime. We used two breeds of mice, which differed in pigmentation (albino versus agouti) and in the loci of glucose phosphate isomerase (Gpi- $1^{a}$ 1 $^{a}$ versus Gpi$\left.1^{b} 1^{b}\right)$. Albino mice (BAMIZ) originated from an outbred colony, established in our laboratory by crossing BALB/C and MIZ mice and selected for Gpi- $1^{a}$ homozygosity. Pigmented mice were F1(C57BL/10 $\times$ CBA/H or a reverse cross) and were Gpi-1b $1^{b}$. Two-to-five month old cycling females were caged with males of the same breed in the evening and inspected for plugs in the next morning (the day of plug = the first day of pregnancy).

\section{Retrieval and manipulation of blastocysts}

The plugged females were sacrificed on the 4 th day after vaginal plug (BAMIZ: between 9.30 and 16.00, F1: between 10.00 and 14.30). Embryos were flushed from the uteri with phosphate buffered saline (PBS; Biomed, Poland) or with M2 medium (Fulton and Whittingham, 1978). The recovered blastocysts were in various stages of expansion and only those with medium-to-large cavity and the expanded blastocysts were selected for fusion. After removal of the zona pellucida in $0.5 \%$ pronase (Sigma) in Ringer solution, blastocysts awaited electrofusion in M2 medium under liquid paraffin at $37^{\circ} \mathrm{C}$ in an atmosphere of $5 \% \mathrm{CO}_{2}$ in air. With the use of phytohemagglutinin (Sigma) $(300 \mu \mathrm{g} / \mathrm{ml}$ in BSA-free $\mathrm{M} 2$ medium) one BAMIZ blastocyst was agglutinated with one $F 1$ blastocyst in agar-coated embryological watch glass. The paired blastocysts were usually at similar stage of expansion. The two embryos were stuck together in the region of the inner cell mass in different spatial configurations, as illustrated in Fig.1: their embryonic-abembryonic axes were parallel $(A)$, oblique $(B)$, perpendicular (C) or reverse (D) to each other. Between 14.00 and 19.00, agglutinated pairs were subjected to electric pulses, according to the modified technique of Kubiak and Tarkowski (1985), in the fusion chamber filled with $0.25 \mathrm{M}$ glucose supplemented with $100 \mu \mathrm{M} \mathrm{CaCl}_{2} \cdot \mathrm{H}_{2} \mathrm{O}$ and $100 \mu \mathrm{M} \mathrm{MgSO}_{4} \cdot 7 \mathrm{H}_{2} \mathrm{O}$, in which the distance between electrodes varied between 240 and $290 \mu \mathrm{m}$. Blastocysts were orientated with their surface of adherence lying perpendicularly to the lines of the electric field and subjected twice to two pulses of 40 Volts d.c. of $25 \mu$ s duration (if required the orientation of a pair was corrected after the first series of pulses). Electrostimulated pairs of blastocysts were cultured in separate drops of KSOM medium (Erbach et al., 1994) (Specialty Media, USA) overnight and inspected for fusion next morning. Blastocysts with a single ICM (formed by incorporation of two ICMs of different origin and thus considered to be chimaeric) were used for transfer to foster mothers.

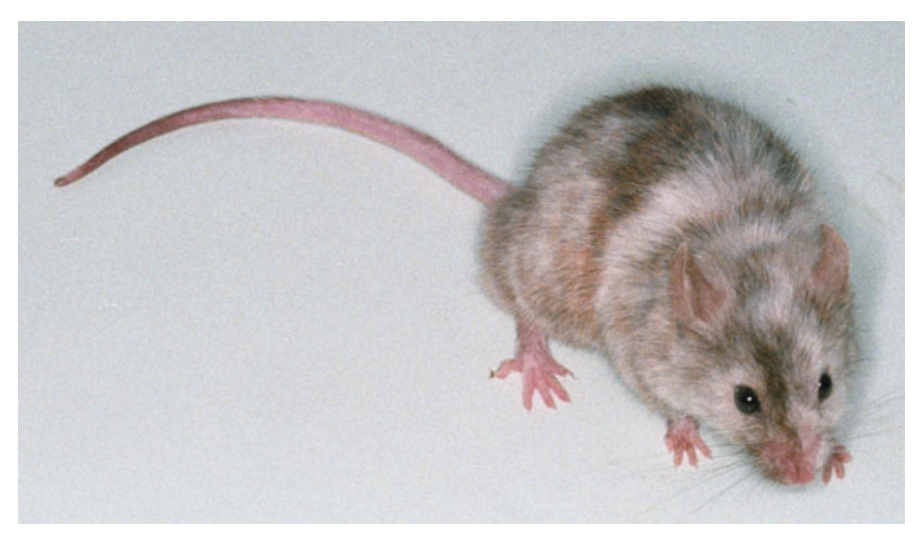

Fig. 5. An adult hermaphrodite chimaera (11 months old). Pigmented areas predominate but albino patches are visible along the whole body.

\section{Transfer of chimaeric blastocysts}

Experimental blastocysts were transferred on the 4th day of pseudopregnancy to the uterus of recipient females. The recipients were MIZ and BAMIZ females (age: 4.5-6 months) mated with vasectomized F1 males. Females were anaesthesized with $0.35 \mathrm{ml}$ of Nembutal (Serva) (6 $\mathrm{mg} / \mathrm{ml}$ distilled water) injected intraperitoneally.

\section{Monitoring of pregnancy}

Starting from the 10th day after mating, the vaginal smears were taken daily to determine whether implantation had taken place and the pregnancy was continued. Females showing signs of proestrus or oestrus were sacrificed and the uterus was inspected for resorptions. Other females were sacrificed on the 20th day. Young, recovered by Caesarian section, were fostered by females that had littered the same night or 24 h earlier. Animals successfully reared were tested for fertility by crossing with normal females. After birth of few litters experimental animals were sacrificed for GPI analysis.

\section{GPI assay}

Samples of tissues/organs were frozen in small amount of redistilled water and stored at $-20^{\circ} \mathrm{C}$. After thawing, the samples were homogenized mechanically, centrifuged at $14000 \mathrm{rpm}$ for $10 \mathrm{~min}$ and the supernatants were collected. Electrophoresis was carried out on TITAN III-H plates (Helena BioSciences, UK) according to Buehr and McLaren (1985). Plates were photographed at different times of staining. From 9 to 13 organs/tissues were examined for each experimental young or adult.

\section{Labelling of embryos with fluorescent dextrans}

Fertilized eggs were obtained from spontaneously ovulating 2-6 monthold $\mathrm{F} 1$ (C57Bl/10 $\times \mathrm{CBA} / \mathrm{H}$ or a reverse cross) plugged females mated to F1 males. Females were sacrificed at noon on the first day of pregnancy. After removing cumulus cells with hyaluronidase (Sigma; $500 \mu \mathrm{g} / \mathrm{ml}$ in $\mathrm{Ca}^{2+} / \mathrm{Mg}^{2+}$-free PBS) eggs were cultured in M2 medium. In the early afternoon, they were injected with a solution of dextran conjugated with fluoresceine or rhodamine (70000 MW; Molecular Probes, USA), at the final concentration of $2 \mathrm{mg} / \mathrm{ml}$ in 2mM PIPES buffer ( $\mathrm{pH}$ 7.4) (Sigma) supplemented with $0.140 \mathrm{mM} \mathrm{KCl}$ (Sigma). About $2 \mathrm{pl}$ of dextran solution was injected with Eppendorf pneumatic microinjector (type 5242). Injected eggs were transferred separately on the day of plug to the oviduct of recipient MIZ females mated with vasectomized F1 males. Seventy two hours after the transfer, the embryos recovered as blastocysts, were aggregated in pairs (partners labelled with different dextrans), electrofused as described above and cultured overnight in KSOM medium under standard conditions. Fused blastocysts were fixed for $20 \mathrm{~min}$ in $2 \%$ paraformaldehyde in $\mathrm{Ca}^{2+} / \mathrm{Mg}^{2+}$-free PBS, $\mathrm{pH} 7.4$ (Biomed, Poland), 


\section{A.K. Tarkowski et al.}

stained for $10 \mathrm{~min}$ at $37^{\circ} \mathrm{C}$ in fluorescent chromatin-specific dye DRAQ5 (Biostatus Ltd.) (10 $\mu \mathrm{M}$ in PBS) and transferred to droplets of PBS in the viewing chamber. Blastocysts were examined with confocal laser Zeiss microscope LSM 510.

\section{Acknowledgements}

This work was partly financed by a grant from the State Committee for Scientific Research (Grant no. 3 P04C 074 22). We wish to thank Professor Marek Maleszewski and Dr. Ewa Borsuk for injecting eggs with fluorescent dextrans and Mr. Darek Maluchnik for assistance with confocal microscopy and for preparation of figures. We are most thankful to Professor Malgorzata Kloc for improving the final version of the manuscript.

\section{References}

BUEHR, M. and McLAREN, A. (1985). Expression of glucose-phosphate isomerase in relation to growth of the mouse oocyte in vivo and in vitro. Gamete Res. 11: 271-281.

ERBACH, G.T., LAWITTS, J.A., PAPAIOANNOU, V.E. and BIGGERS, J.D. (1994). Differential growth of the mouse preimplantation embryo in chemically defined media. Biol. Reprod. 50: 1027-1033.

FALCONER, D.S. and AVERY, P.J. (1978). Variability of chimaeras and mosaics. J. Embryol. Exp. Morph. 43: 195-219.

FULTON, B.P. and WHITTINGHAM, D.G. (1978). Activation of mammalian oocytes by intracellular injection of calcium. Nature 273: 149-151.

GARDNER, R.L. (1971). Manipulations on the blastocyst. Advances in Biosciences 6: $279-296$

GARDNER, R.L. (1997). The early blastocyst is bilaterally symmetrical and its axis of symmetry is aligned with the animal-vegetal axis of the zygote in the mouse. Development 124: 289-301.

GARDNER, R. L. and JOHNSON, M. H. (1973). Investigation of early mammalian development using interspecific chimaeras between rat and mouse. Nature New Biol. 246: 86-89.

KUBIAK, J. Z. and TARKOWSKI, A.K. (1985). Electrofusion of mouse blastomeres. Exp. Cell Res. 157: 561-566.

McLAREN, A. (1976). Mammalian chimaeras. Cambridge University Press, Cam- bridge, London, NewYork, Melbourne.

McLAREN, A. (1984). Chimeras and sexual differentiation. In Chimeras in Developmental Biology (Eds. N. Le Douarin and A. McLaren). Academic Press, London, Orlando, San Diego, New York, Toronto, Montreal, Sydney, Tokyo, pp 381-399.

MULLEN, R.J. and WHITTEN, W.K. (1971). Relationship of genotype and degree of chimerism in coat color to sex ratios and gametogenesis in chimeric mice. $J$. Exp. Zool. 178: 165-176.

MYSTKOWSKA, E.T. and TARKOWSKI, A.K. (1968). Observations on CBA-p/ CBA-T6T6 mouse chimeras. J. Embryol. Exp. Morph. 20: 33-52.

MYSTKOWSKA, E.T., OZDZENSKI, W. and NIEMIERKO, A. (1979). Factors regulating the degree and extent of experimental chimaerism in the mouse. $J$. Embryol. Exp. Morph. 51: 217-225.

OZIL, J. P. (1983). Production of identical twins by bisection of blastocysts in the cow. J. Reprod. Fertil. 69: 463-468.

OZDZENSKI, W., SZCZESNY, E. and TARKOWSKI, A.K. (1997). Postimplantation development of mouse blastocysts with two separate inner cell masses. Anat. Embryol. 195: 467-471.

SKRZYSZOWSKA, M., SMORAG, Z., KATSKA, L. and BOCHENEK, M. (1999). Cattle twins after transfer of demi-embryos derived from zona-perforated blastocysts. J. Anim. Feed Sci. 8: 223-231.

SMITH, L.J. (1980). Embryonic axis orientation in the mouse and its correlation with blastocyst relationships to the uterus. I. Relationships between 82 hours and $4^{1 / 4}$ days. J. Embryol. Exp. Morph. 55: 257-277.

SMITH, L.J. (1985). Embryonic axis orientation in the mouse and its correlation with blastocyst relationships to the uterus. II. Relationships from $4^{1 / 4}$ to $9^{1 / 2}$ days. J. Embryol. Exp. Morph. 89: 15-35.

TARKOWSKI, A.K. (1961). Mouse chimaeras developed from fused eggs. Nature 190: 857-860.

TARKOWSKI, A.K. and WOJEWODZKA, M. (1982). A method for obtaining chimaeric mouse blastocysts with two separate inner cell masses: a preliminary report. J. Embryol. Exp. Morph. 71: 215-221.

Received: April 2005

Reviewed by Referees: May 2005

Modified by Authors and Accepted for Publication: August 2005 